Supplementary Information

\title{
Highly Chiroptical Detection with Gold-silver Bimetallic Nanoclusters Circularly Polarized Luminescence Based on G-quartet Nanofiber Self-assembly
}

Zhiguang Suo, ${ }^{a}$ Xialing Hou, ${ }^{a}$ Jingqi Chen,${ }^{a}$ Xiaowei Liu, ${ }^{a}$ Yu Liu, ${ }^{b}$ Feifei Xing, ${ }^{c}$ Yingying Chen ${ }^{a}$ and Lingyan Feng, ${ }^{* a}$

${ }^{a}$ Materials Genome Institute, Shanghai University, Shanghai 200444, P. R. China.

${ }^{b}$ Qianweichang college, Shanghai University, Shanghai 200444, P. R. China.

${ }^{c}$ Department of Chemistry, College of Science, Shanghai University, Shanghai 200444, P. R. China.

*Corresponding author: lingyanfeng@t.shu.edu.cn 


\section{Experimental Details}

The UV-vis absorption spectra were performed on a Jasco J-750 spectrophotometer, and characterized in $1.0 \times 1.0 \mathrm{~cm}$ quartz cuvette. An Edinburgh FS5 fluorescence spectrometer was performed for fluorescence spectroscopy measurements. The spectrum correction was selected at the slits of $2 / 2 \mathrm{~nm}$. Life-times were obtained from the decay curves by following the equation: $R(t)=\sum_{i} B_{i} e^{-t / \tau_{i}}\left(\chi^{2}<1.2\right)\left(B_{i}\right.$ is the amplitude of the $i^{t h}$ component at time $t$, and $\tau_{i}$ is the lifetime of the $i^{t h}$ ) using Edinburgh TCSPC (time-correlated single photon counting, excited at $365 \mathrm{~nm}$ ) on an Edinburgh Fluoracle analytical program instruments. The average lifetime $\left(\tau_{a v}\right)$ was fitted according to the equation: $\tau_{a v}=\left(\sum \alpha_{i} \tau_{i}^{2}\right) /\left(\sum \alpha_{i} \tau_{i}\right)\left(\alpha_{i}\right.$ represent the weights of the components $\tau_{i}$ ). Quantum yield (QY) measurement of Au NCs and AuAg NCs were performed using an Edinburgh FS-5 UK fluorescence spectrometer by an absolute method. The QY was carried out by direct excitation in the integrating sphere. And, the final calculation of QY is supplied with the Fluoracle software according to:

$$
Q Y=\frac{N^{e m}}{N^{a b s}}=\frac{E_{b}-E_{a}}{S_{a}-S_{b}}
$$

where $N^{a b s}$ and $N^{e m}$ are the amount of photons absorbed and emitted respectively. $E_{a}$ and $E_{b}$ are the amount of photons emitted of reference and sample. $S_{a}$ and $S_{b}$ are the amount of photons absorbed of reference and sample. Far-UV circular dichroism (CD) spectra were characterized using $4.0 \times 10 \mathrm{~mm}$ quartz cuvette in a Jasco J-1500 spectropolarimeter under a nitrogen atmosphere. And CPL spectra measurement was recorded on Jasco CPL-300 spectrometer. The sizes and morphologies of AuAg NCs were obtained by using Hitachi HT7700 transmission electron microscope. The diluted AuAg NCs sample was deposited on a carbon support copper grid and air-drying. The morphology of the g-fiber was characterized using field-emission scanning electron microscope (SEM, Carl Zeiss G300). X-ray photoelectron spectroscopy (XPS) measurements were carried out on Escalab 250xi spectrometer. The fluorescent images of R-/L-hand chiroptical materials were taken on an Olympus FV3000 confocal microscope. Fourier transform infrared (FT-IR) spectra were 
obtained on a Nicolet 6700 spectrometer (Thermo). All measurements were performed three times to obtain more reliable results.

The synthesis of Au NCs using different ligands is shown below. Bovine Serum Albumin (BSA) templated Au NCs were synthesized by one-pot method. Briefly, $2.0 \mathrm{~mL} \mathrm{HAuCl}{ }_{4}(10$ $\mathrm{mM})$ was add into BSA solution $(50 \mathrm{mg} / \mathrm{mL})$. Then $0.1 \mathrm{ml} \mathrm{NaOH}(1.0 \mathrm{M})$ solution was injected into mixture under vigorous stirring at $37{ }^{\circ} \mathrm{C}$ for $24 \mathrm{~h} .{ }^{1} \mathrm{In}$ a typical procedure of histidine-mediated AuNCs, an aqueous solution of $\mathrm{HAuCl}_{4}(1 \mathrm{ml}, 10 \mathrm{mM})$ was blended with histidine $(3 \mathrm{ml}, 0.15 \mathrm{M})$ at room temperature for $2 \mathrm{~h}^{2}{ }^{2}$ The glutathione (GSH) stabilized $\mathrm{Au}$ NCs were prepared according the literature with a little modification. In brief, HAuCl4 solution $(20 \mathrm{mM}, 0.4 \mathrm{~mL})$ was added into $\mathrm{GSH}$ solution $(40 \mathrm{mM}, 0.3 \mathrm{~mL})$ under vigorous stirring over 10 minutes. After the mixture turned clear, $\mathrm{H}_{2} \mathrm{O}(4.3 \mathrm{~mL})$ was added into the mixture. And, the mixture was heated at $70{ }^{\circ} \mathrm{C}$ under stirring for $12 \mathrm{~h} .{ }^{3}$ 

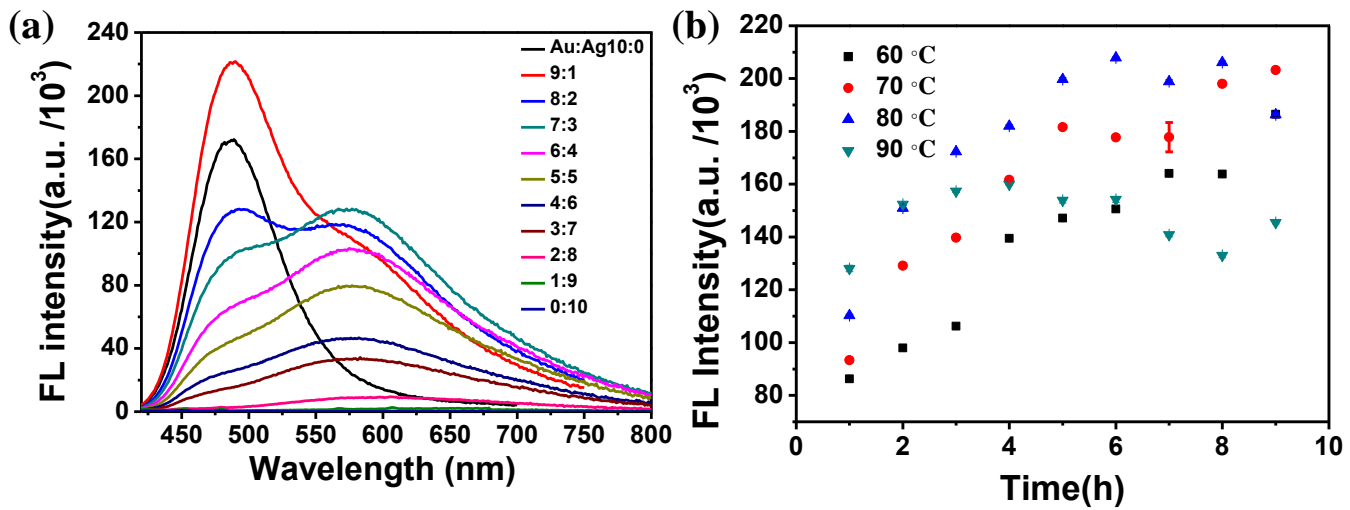

Figure S1. Emission spectra of aqueous solutions of AMP-AuAg NCs at different ratio of $\mathrm{Au}$ and $\mathrm{Ag}(\mathrm{a})$, reaction time and temperature (b).
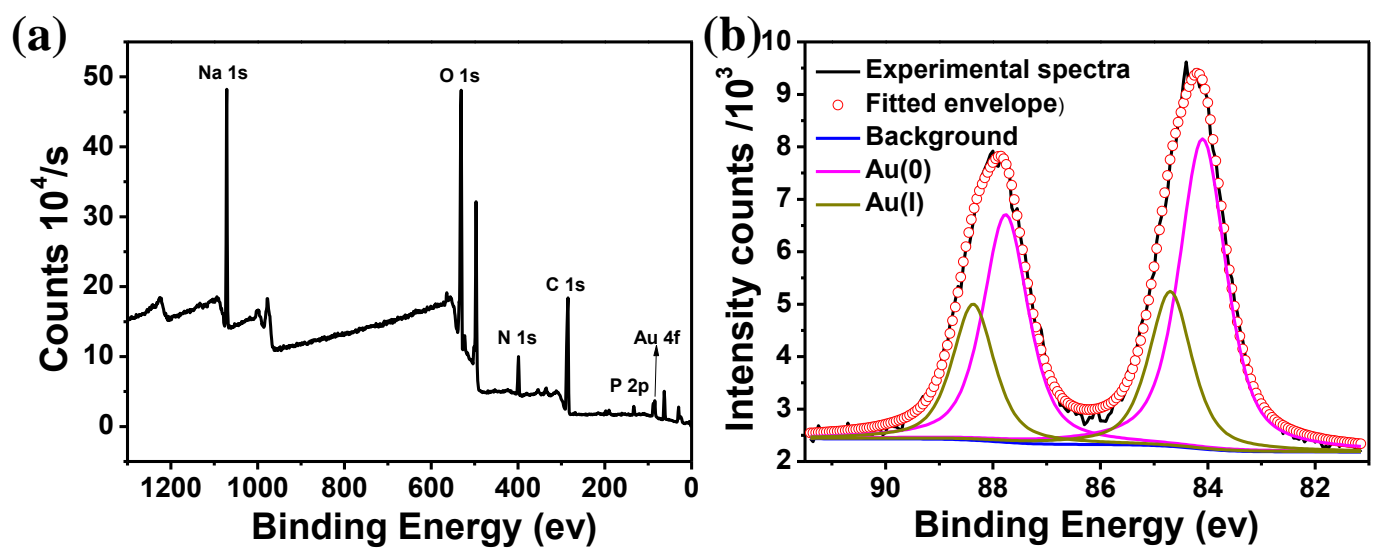

Figure S2. The XPS spectra of the AMP-Au NCs: (a) survey scan; (b) Au 4f.
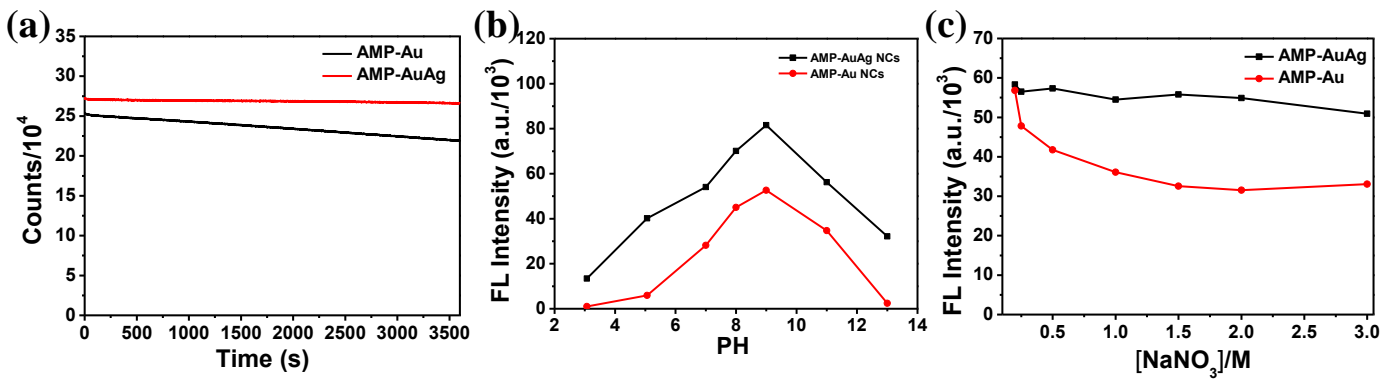

Figure S3. (a) Time response of fluorescence of AMP-Au NCs and AMP-AuAg NCs within 1h. (b) The Fluorescence changes of AMP-Au NCs and AMP-AuAg NCs at different pH. (c) Fluorescence intensity of AMP-Au NCs and AMP-AuAg NCs in solution over a salt concentrations of $0.2 \mathrm{M}$ to $3 \mathrm{M} \mathrm{NaNO}$. 


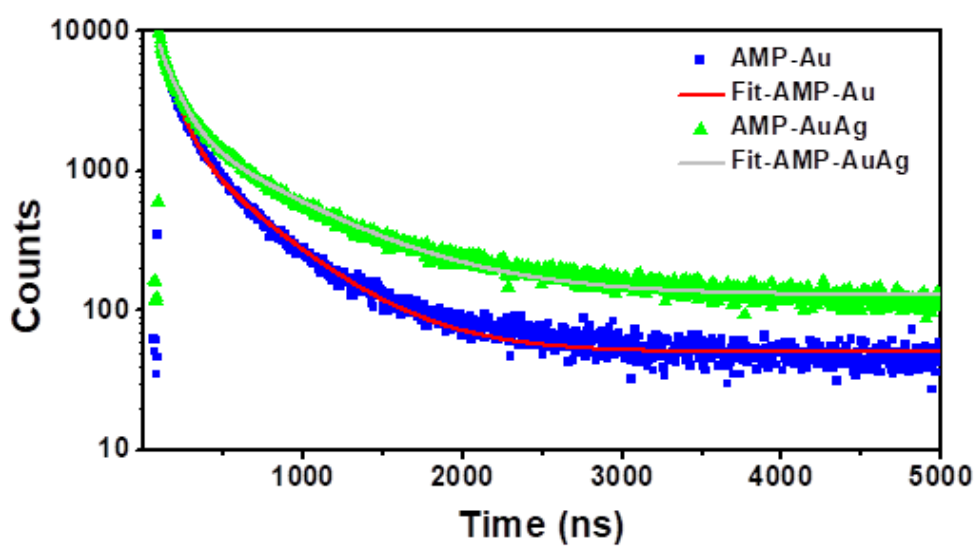

Figure. S4. Fluorescence decays of AMP-AuAg NCs and AMP-Au NCs .

Table S1. Fluorescent lifetime and quantum yield comparison of AMP-Au NCs and AMP-AuAg NCs.

\begin{tabular}{lcccccc}
\hline & $\boldsymbol{\tau}_{\mathbf{1}}(\mathrm{ns})$ & $\boldsymbol{\alpha}_{\mathbf{1}}(\boldsymbol{\%})$ & $\boldsymbol{\tau}_{\mathbf{2}}(\mathrm{ns})$ & $\boldsymbol{\alpha}_{\mathbf{2}}(\boldsymbol{\%})$ & $\boldsymbol{\tau}_{\mathrm{av}}(\mathrm{ns})$ & $\mathrm{QY}(\%)$ \\
\hline AMP-Au NCs & 4572.69 & 41.11 & 1594.11 & $58.89 \%$ & 3580.64 & 3.67 \\
\hline $\begin{array}{c}\text { AMP-AuAg } \\
\text { NCs }\end{array}$ & 5138.25 & 35.64 & 1763.94 & 64.36 & 3846.93 & 9.42 \\
\hline
\end{tabular}
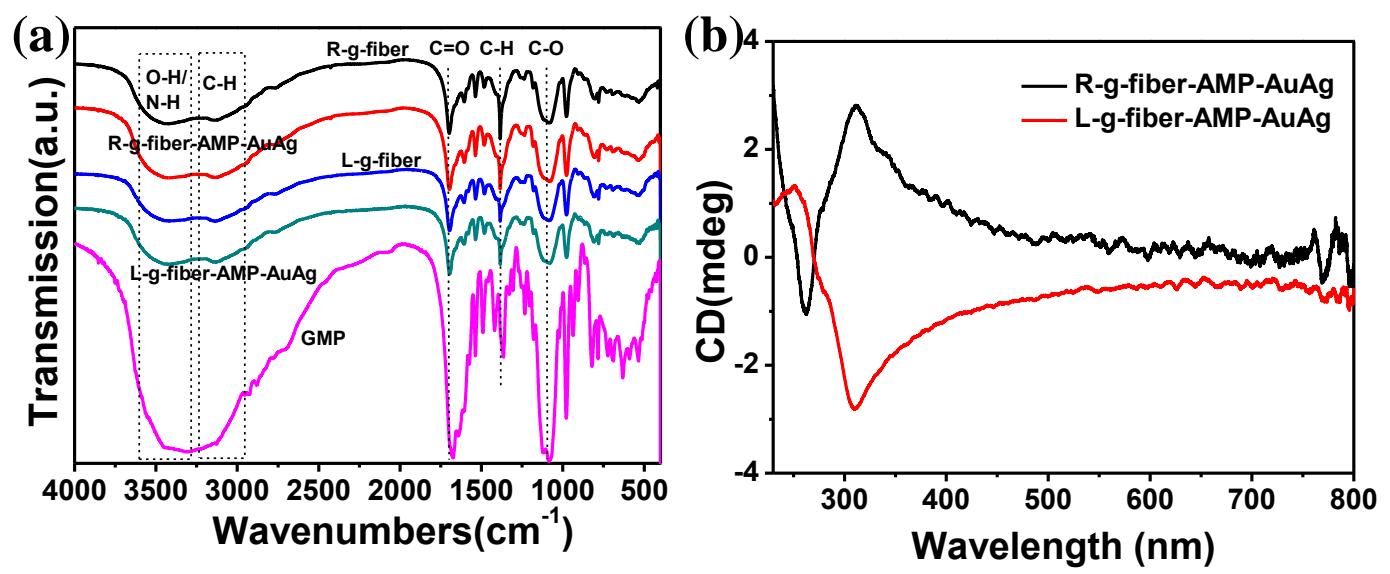

Figure S5. (a) FT-IR spectra of g-fiber and assembled with AuAg NCs. (b) Far-UV circular dichroism (CD) spectra of R-/L- g-fiber assembled with AuAg NCs.. 

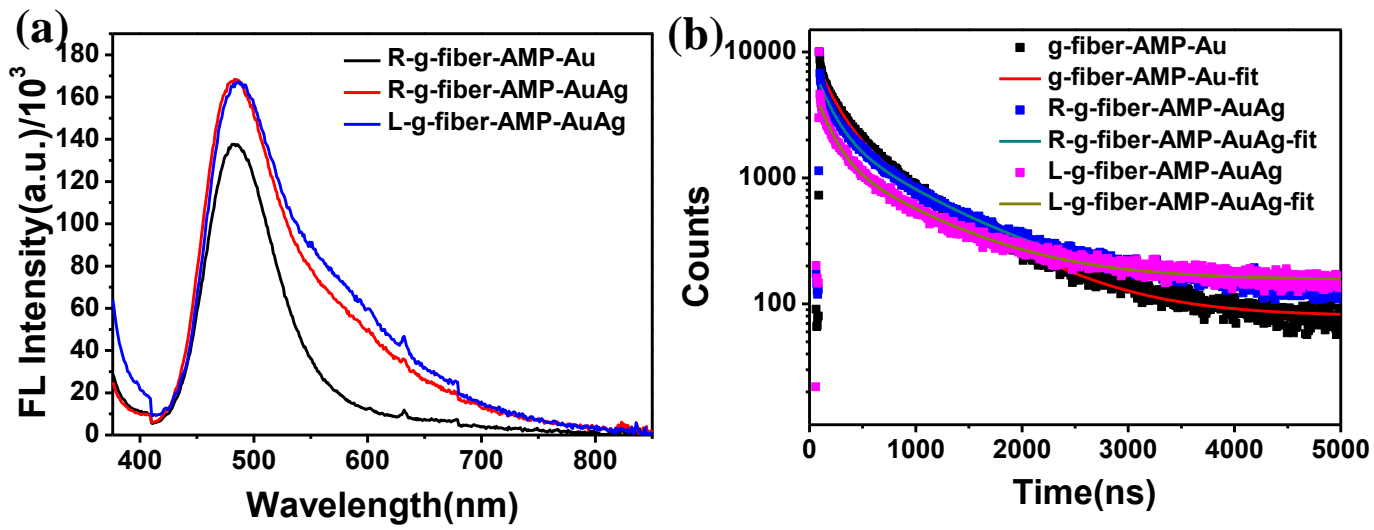

Figure. S6. (a) Fluorescence emission spectra of R-/L- g-fiber-AuAg NCs compared with g-fiber-Au NCs. (b) Fluorescence decays of R-/L- g-fiber-AMP-AuAg NCs.

Table S2. Fluorescent lifetime comparison of the assembled R-/L- g-fiber-AuAg NCs with g-fiber-Au NCs.

\begin{tabular}{cccccc}
\hline & $\boldsymbol{\tau}_{\mathbf{1}}(\mathrm{ns})$ & $\boldsymbol{\alpha}_{\mathbf{1}}(\boldsymbol{\%})$ & $\boldsymbol{\tau}_{\mathbf{2}}(\mathbf{n s})$ & $\boldsymbol{\alpha}_{\mathbf{2}}(\boldsymbol{\%})$ & $\boldsymbol{\tau}_{\mathrm{av}}(\mathrm{ns})$ \\
\hline g-fiber-AMP-Au & 207 & 30.7 & 784.92 & 69.3 & 724.46 \\
\hline R-g-fiber-AMP-AuAg & 209.38 & 26.25 & 916.45 & 73.75 & 863.28 \\
\hline L-g-fiber-AMP-AuAg & 180.18 & 26.55 & 859.39 & 73.45 & 811.54 \\
\hline
\end{tabular}

\section{Reference}

(1) Xie, J.; Zheng, Y.; Ying, J. Protein-directed Synthesis of Highly Fluorescent Gold Nanoclusters. J. Am. Chem. Soc. 2009, 131, 888-889

(2) Guo, Y.; Zhao, X.; Long, T.; Lin, M.; Liu, Z.; Huang, C. Histidine-mediated Synthesis of Chiral Fluorescence Gold Nanoclusters: Insight Into the Origin of Nanoscale Chirality. RSC Adv. 2015, 75, 61449-61454.

(3) Tang, Y.; Xu, J.; Xiong, C.; Xiao, Y.; Zhang, X.; Wang, S. Enhanced Electrochemiluminescence of Gold Nanoclusters via Silver Doping and Their Application for Ultrasensitive Detection of Dopamine. Analyst 2019, 144, 2643-2648. 